\title{
Dynamic Game Research on Asset Structure Optimization
}

\author{
CHEN He1, a, LIU Baifang2, b, SUI Liqiü, c
}

\author{
1Business School, Beijing Language and Culture University, Beijing, 100083, China \\ 2Business School, Beijing Language and Culture University, Beijing, 100083, China \\ ${ }^{3}$ School of Accountancy, China Central University of Finance and Economics, Beijing,100081, \\ China
}

aemail: chenhe@blcu.edu.cn, bemail: liubaifang@blcu.edu.cn, cemail:suiliqiu@yeah.net

Keywords: Asset Structure Optimization; Path Dependence; Dynamic Game Model

\begin{abstract}
Through constructing complete information dynamic game model, this paper studies the path dependence and dynamic game of enterprise asset structure optimization. Asset structure optimization strategy path depends on the expected asset structure and product market competition strategy. In the long run, the path of the asset structure optimization strategy is unstable. The change of the capital contract, the change of the transformation factor and the change of managers' cognition of equilibrium return lead to the inconsistency of the asset structure optimization strategy.
\end{abstract}

\section{Introduction}

In this paper a new optimal design of soccer robot control system which is based on mechanical analyses and calculations on the pressure and transmutation states of chip kick mechanics, this new control system with high precision for speed control and high dynamic quality.North pointed out that the system in formation and evolution has the character of path dependence in the analysis of the relationship between institution, institutional change and economic performance.

\section{Game Model}

The participants of the game are the managers and investors of the enterprise. The investors choose the expected corporate assets structure, that is, the expected asset structure optimization strategy taken by managers; meanwhile, the investors can adjust the corporate asset structure in accordance with the path of capital contract to ensure the expected asset structure by the construction of reasonable capital contract. Managers are confronted with the constraints of capital contracts based on the investors' expected asset structure to select the actual optimization strategy and determine the actual corporate asset structure.

\section{Utility Function}

We assume investors' expected asset structure is $\mathrm{A}^{\mathrm{e}}$, and the manager's actual-optimized asset structure is A. Based on the behavior of the managers' optimizing asset structure driven by the capital contract and the competition, it brings utility of two aspects: one is the optimization of the asset structure; the other is the change of the company's income resulting from the optimization of the asset structure. We assume that the utility of the company manager is a function of the asset structure and the company's return. If the actual yield of the company's product market is $i$, when there is a perfect capital market, the product market equilibrium rate of return is $i_{0}$. If the manager is risk- averse, the manager's asset structure optimization function is as follows:

$$
U(A, i)=-k A^{2}-\left(i-l i_{0}\right)^{2}, 0 \leq k \leq 1, l>1
$$

Where $-k A^{2}$ fitting the driving incentives that manager's driven by the capital contract to 
optimize the allocation of corporate initial asset structure and to explore the optimal asset structure. In the long run, the incentive that managers have been driven by capital contracts is changed.

In the single period of the asset structure optimization, the conversion factor is $\beta$ in the constraint function of the enterprise income, that is, the revenue generated by the manager's asset structure optimization strategy. During the current period of asset structure optimization, the manager's asset structure optimization strategy may not develop the enterprise's yield, at this time, $\beta=0$; on the contrary, during the current period of asset structure optimization, the manager's asset structure optimization strategy fully achieves the enterprise's current yield, at this time, $\beta=1$. When $\beta \in(0,1)$, the enterprise' yield brought by managers' asset structure optimization strategy distributes in each period. With the value of $\beta$ getting larger, the distribution of corporate yield emphasize on early periods.

Equilibrium Strategy

The game model in the former part is asset structure expectation of given investors, and the managers choose the asset structure optimization strategy. Considering the single game, the manager is to weigh the problem:

$$
\operatorname{Max} U(A, i)=-k A^{2}-\left(i-l i_{0}\right)^{2}, k>0, l>1 \text {, s.t. } i=i_{0}+\beta\left(A-A^{e}\right), 0 \leq \beta \leq 1
$$

We use first-order condition to solve the optimization problem above. If we set $\frac{\partial \operatorname{Max} U(A, i)}{\partial A}=0$, the equilibrium solution of the managers' single-period optimal strategy of the asset structure is obtained: $A^{*}=\beta\left[\beta A^{e}+(l-1) i_{0}\right] /\left(k+\beta^{2}\right)$

Equilibrium result for single complete information dynamic asset structure optimization game is $\left(A^{*}, A^{e}\right)$. The equilibrium solution of the managers' single-period optimal strategy of the asset structure is obtained: $A^{*}=\frac{\beta^{2} A^{e}}{k+\beta^{2}}+\frac{\beta(l-1)}{k+\beta^{2}} i_{0}, 0 \leq \beta \leq 1$

Obviously, the response function of the managers' asset structure optimization is composed of two parts: the investors' expected asset structure and equilibrium return determined by the market competition. $\beta^{2} /\left(k+\beta^{2}\right)$ and $\beta(l-1) /\left(k+\beta^{2}\right)$ are respectively the sensitive coefficients of optimal asset structure for investors' expectation of asset structure and equilibrium return.

\section{Explanation of Equilibrium Strategy}

The response function of the manager's asset structure optimization strategy describes the path dependent characteristics of the enterprise's optimal asset structure. Asset structure optimization path depends on the expected asset structure and equilibrium return rate of investors.

First of all, investors' expectation is taken into analysis. In the previous paper, we assume the expected asset structure is known, so $A^{e}$ is fixed. The manager's assets structure optimization strategy is adjusted based on the investors' expected asset structure $A^{e}$, and the adjustment coefficient is $\beta^{2} /\left(k+\beta^{2}\right)$. Path dependent parameter $\mathrm{k}$ measures the extent to which the managers are driven by capital contracts and optimize the structure of assets in accordance with the requirements of capital contracts. In the single-period game, the $\mathrm{K}$ value is constant, but the $\mathrm{K}$ value is changed in the multi-period game.

Second, we analyze the impact of the equilibrium return. The mechanism of equilibrium returns to the management of the asset structure optimization strategy is not only affected by the conversion factor of the optimal strategy, ,but also by the path dependence parameters of asset structure optimization,k, how managers deal with the constraint of capital contracts. From reaction function of the management of the asset structure optimization, it's obvious that the equilibrium rate of return acted on the asset structure optimization strategy choices through the coefficient $\beta(l-1) /\left(k+\beta^{2}\right)$, where, (l-1) demonstrates the degree of distortion between the risk and information asymmetry to the equilibrium rate of return. In a single game, it is reasonable to 
assume that the manager thinks the market distortion degree is a fixed value. In the continuous game model, the manager can modify the degree of distortion of the market. Therefore, $(l-1)$ in the multi-period game is varied. The manager's correction of market distortions can lead to inconsistencies in asset structure optimization paths. Regarding this, we will take it into deeper analysis in the latter part.

Through the above analysis, it is clear that the managers' path of the asset structure optimization strategy depends on the investors' expected asset structure and equilibrium return rate. When the capital structure of the firm is fixed, the managers' evaluation of the conversion factor, $\beta$, has a complex mechanism for the asset structure optimization.

\section{Dynamic Inconsistency}

The path dependence parameter $\mathrm{k}$ and $\beta$ are varied in the dynamic game, which fits the dynamic inconsistency of the asset structure optimization path. Varied path dependence parameters reflect the shift of the investor the expected weight of the asset structure and the equilibrium return rate that manager's asset structure optimization strategy gives.

\section{Influence of Path Dependence Parameter Variation}

First, when the $\mathrm{K}$ value changes, the role of capital contracts to the managers' asset structure optimization path limits, which leads to the dynamic inconsistency of asset structure optimization path. Assuming $\beta$ is a constant, as the $\mathrm{k}$ value increases, the proportion of the asset structure of the investors is expected to be reduced, and the $\mathrm{A}^{*}$ deviation $A^{e}$ from increases. In other words, with the manager's increasing incentives to adjust the asset structure, the investors' expected asset structure reduces the path constraint of managers' asset optimization strategy. In determining asset structure optimization strategy, managers may break through the path of the asset structure optimization strategy, thus the dynamic inconsistency of the optimal strategy appears.

Secondly, with the increase of $\mathrm{K}$ value, the proportion of equilibrium return the managers' asset structure optimization strategy gives is increasingly small. The management of the optimization strategy emphasize more on the capital structure, market competition strategy or the managers' own utility and other contractual factors. With regard to K, the mechanisms of investors' expected asset structure and expected equilibrium return are the same. However, when the conversion factor $X$ is introduced, the mechanism of the effect of $K$ on the equilibrium yield becomes complex. If $\beta<\sqrt{k}<1$, with the increase of $\beta$ value, when managers determine the optimal strategy of asset structure, the proportion of equilibrium is increasing; if $\sqrt{k}<\beta$, with the increase of $\beta$ value, when managers determine the optimal strategy of asset structure, the proportion of equilibrium is decreasing.

Again, the special path dependence parameter points include two points: the endpoint and the extreme point. It is known from the analysis of the coefficient of equilibrium yield that the mechanism of the effect on the equilibrium return depends on the actual value. When $0<k<1$, the effect of the conversion factor $\beta$ on the market equilibrium return is not monotonous. When $\mathrm{k}$ takes 0 or 1 , the conversion factor $\beta$ has a monotonous effect on the equilibrium return. Because of the complex relationship between path dependent parameters, we must consider the investors' expected asset structure and equilibrium return rate when analyzing the endpoint solution of $\mathrm{k}$ and $\beta$.

\section{Refined Equilibrium Strategy}

When $\beta=\sqrt{k}, 0<k<1$, the impact of the conversion factor $\beta$ on the equilibrium return reached the peak value, and the weight the equilibrium return the manager gives reaches maximum value. When $\beta=\sqrt{k}=1$, the weight the equilibrium return the manager gives can also reach maximum value. It is known from the reaction function $A^{*}$ that managers must also weigh the impact of expected asset structure and equilibrium yield to determine asset structure optimization strategy. If 
we assume, the managers' optimal response strategy of the asset structure is $A_{2}^{*}=\frac{1}{2}\left[A^{e}+\frac{l-1}{\sqrt{k}} i_{0}\right]$,

If we assume, the managers' reaction function of optimal response strategy is: $A_{2}^{*}=\frac{1}{2}\left[A^{e}+\frac{l-1}{\sqrt{k}} i_{0}\right]$.From the comparison between $A_{1}^{*}$ and $A_{2}^{*}$, we can get $A_{1}^{*}=\frac{1}{2}\left[A^{e}+(l-1) i_{0}\right]<A_{2}^{*}=\frac{1}{2}\left[A^{e}+\frac{l-1}{\sqrt{k}} i_{0}\right]$,

So, when $\beta=\sqrt{k}, 0<k<1$, the managers' optimal asset structure strategy is

$A_{2}^{*}=\frac{1}{2}\left[A^{e}+\frac{l-1}{\sqrt{k}} i_{0}\right], 0<k<1$ 。

The managers' optimal asset structure optimization strategy $A_{2}^{*}$ is the result of refining of the managers' response function $A^{*}$ which is the equilibrium strategy of single-period asset structure complete information game. At this time, path dependence parameters $\mathrm{k}$ and $\beta$ are fixed. But when mangers and investors conduct multi-period asset structure complete information game, path dependence parameters $\mathrm{k}$ and $\beta$ are varied and there is a delicate relationship between the two parameters. It is $A_{2}^{*}$ that is the re-refining of mangers' equilibrium strategy after taking dynamic change constraint mechanism of path dependence parameters $\mathrm{k}$ and $\beta$. The managers' optimal strategy $A_{1}^{*}$ is the corner solution.

Managers will choose strategy $A_{2}^{*}$ after trading off the delicate relationship between path dependence parameter $\mathrm{k}$ and $\beta$. Strategy $A_{2}^{*}$ is the weighted average number of investors' expected capital structure and equilibrium return. Strategy $A_{2}^{*}$ gives the equilibrium return of product market a weight of $\frac{l-1}{2 \sqrt{k}}$, which demonstrates that the capital contract of the enterprise exerts influence on the product market strategy of the enterprise through managers' giving weight to the equilibrium return and finally shifts the corporate value. In the single-period game, the strategy $A_{2}^{*}$ shows that the path of the manager's optimization strategy depends on the capital contract and the equilibrium return. In dynamic game, the strategy $A_{2}^{*}$ is not stable. As the analysis of strategy $A_{1}^{*}$ this paper points out, the investors always have the desire to use the contract to encourage the managers to choose the corner point $A_{1}^{*}$, and the corner point $A_{1}^{*}$ is not the optimal strategy for the manager. The constraint of the capital contract to the manager is changed in the dynamic game. The change of such constraint leads to the fluctuation of manager's asset structure optimization strategy in the first-period optimal strategy $A_{2}^{*}$ or between the first-period optimal strategy $A_{2}^{*}$ and corner solution $A_{1}^{*}$ in the dynamic game. The stronger the capital contract constraints to the manager are, the closer the actual asset structure optimization strategy is to the corner point $A_{1}^{*}$. The dynamic change of the strategy $A_{2}^{*}$ vividly states the dynamic inconsistency the asset structure optimization path.

\section{Influence of Investor's Rational Expectation}

If the investor is rational, it can reasonably expect the manager's asset structure optimization strategy $\mathrm{A}^{*}$. That is $\mathrm{A}^{*}=\mathrm{A}^{\mathrm{e}}$, put the equation to the managers' optimal strategy reaction function, we can get $A^{*}=A^{e}=\frac{\beta(l-1)}{k} i_{0}$. Left part of the formula states when the investors reasonably expect managers' asset optimization strategy, managers' asset structure strategy path depends on the modified equilibrium return. Its path dependence parameter is $\frac{\beta}{k}$.

The single-period path dependent parameters are fixed, but the parameters are changed in the 
dynamic game. Put the investors' reasonable expectation into the corner solution and refined equilibrium strategies, then we can get: $A_{1}^{*}=(l-1) i_{0}, A_{2}^{*}=\frac{l-1}{\sqrt{k}} i_{0}$

According to the analysis of the "refined equilibrium strategy", in the case of the reasonable expectations of the investors, the refined equilibrium strategy and the corner solution are not stable. As the game is carried out, the managers always deviate from the optimization path, which leads to the dynamic inconsistency of the asset structure optimization strategy.

Influence of Managers' Modified Cognition

In the single stage game model, it is reasonable to assume that the manager's modification of equilibrium is fixed, that is, $l$ is fixed. In the multi-period game model, the manager can "learn during work", summarize experience in time, and improve the modification of the equilibrium return, that is, the $l$ is changed in the multi-period game model. It is known from $\lim _{l \rightarrow 1}(l-1)=0$ that as the product market is closer to the perfect competition market, the manager believes that the market distortion is smaller, whose asset structure optimization strategy gives the less weight to equilibrium return. Managers pay more attention to investors' expected asset structure and capital contracts to optimize the asset structure. It is known from that $\lim _{l \rightarrow \infty}(l-1)=\infty$ as the product market is closer to the monopoly market, the manager believes that the market distortion is larger, whose asset structure optimization strategy gives the more weight to equilibrium return. Managers can get more return from the product market. Dynamic change of $l$ mainly influence on the game of asset structure optimization strategy. In the long run, the managers' delicate adjustment to the equilibrium return can lead to the dynamic inconsistency of the asset structure optimization strategy.

\section{Conclusion}

Based on the complete information dynamic game model of asset structure optimization strategy, the paper studies the path dependent characteristics and the dynamic game of the asset structure optimization strategy of the managers. Through the analysis of the reaction function $\mathrm{A}^{*}$ of the managers in the single-period game, we can get the managers' asset structure optimization strategy path dependent on the expected asset structure and the product market equilibrium return rate. Managers give different weights to both of them.

\section{References}

[1] Aghion P., Dewatripiont M. and Rey P. Renegotiation-design under unverifiable infermation[J]. Lead Article in Econometrica,1994.

[2] Ross. The determination of financial structure: the incentive signaling approach[J]. Bell Journal of Economics, 8,23-40.

[3] Grossman S. and Hart O. Corporate financial structure and managerial incentives[A] Mccall J, The Economics of Information and Uncertainty[C], 1982,Chicgo: University of Chicago Press.

[4] Myers S. and Mailuf N. Corporate financing and investment decisions when the firm has information that investors do not have[J]. Journal of Financial Economics,1984, 13,187-221. 